\title{
Monitoring of agrophysical properties of the active layer of drained long-frozen peat deposits in Western Siberia
}

\author{
Vasily Novokhatin ${ }^{1, *}$, Nadezhda Osipova ${ }^{1}$ \\ ${ }^{1}$ Industrial University of Tyumen, Tyumen, Russia
}

\begin{abstract}
In this study, the authors considered the dynamics of the main agrophysical properties of hydromorphic geosystems in Western Siberia. In the process of evolution, as well as in the conditions of technogenic impact, the soils of natural-territorial complexes, their properties are subject to significant changes. New hydromorphic geosystems are formed, the soils of which are characterized by changed agrophysical properties. Modern technologies for developing investment projects for soil reclamation of hydromorphic geosystems should be based on agrophysical characteristics of reclaimed soils, on the basis of which it is possible to create the necessary parameters of drainage systems and, as a result, provide optimal soil regimes for agricultural crops for a long period. The difficulty is that these soil characteristics are variable over time. Until recently, agrophysical characteristics of soils obtained in other regions of the country were used in the development of reclamation projects for hydromorphic soils in Western Siberia. The attempt to borrow them has led to the fact that large areas of drained soil have become completely unsuitable and to restore their project productivity, a number of agro-reclamation activities and the attraction of large financial resources are currently required. In this regard, there is a need for a comprehensive, long-term study of the main properties of hydromorphic soils used for agricultural purposes, and especially their changes over time under the influence of anthropogenic load.
\end{abstract}

\section{Introduction}

The swamps of Western Siberia contain huge reserves of hydromorphic soils, which make up $30 \%$ of the world's resources [1]. The largest number of them is located in the taiga, subtaiga and forest-steppe subzones. The area of swamps in these subzones is 32.538 million hectares, of which about 5.0 million hectares are located in the agricultural zone. The average depth of peat deposits that can be involved in agricultural turnover varies from $1.7 \mathrm{~m}$ to 2.1 $\mathrm{m}$, respectively, for transitional and low-lying swamps. Of the above varieties, hydromorphic soils of lowland swamps are among the most fertile soils. In terms of nitrogen and organic matter reserves, they are richer than chernozems [2].

In Western Siberia, there were more than 300 thousand hectares of drained lowland marsh geosystems, including about 90 thousand hectares in the Tyumen region. Due to the fact that

* Corresponding author: Novohatin@yandex.ru 
close attention is currently paid to environmental safety in hydromorphic geosystems, the first place is given to the complex use of reclamation measures that maximally "fit" into the natural conditions of swamp landscapes, providing reasonable economic efficiency and environmental sustainability. This will allow you to adjust the rate of change in the agrophysical properties of deposits over time.

\section{Results and discussion}

During 1978-2017, we conducted research on changes in the agrophysical properties of the active layer of drained hydromorphic soils from the standpoint of a systematic approach to assessing their quantitative characteristics, which allows us to reliably assess their changes in time and space. The study covered peat-swamp hydromorphic soils on medium peat in hospitals with atmospheric slope and soil-pressure type of water supply. Laboratory tests were performed using approved methods.

The analysis and generalization of the results of field and laboratory studies were carried out using the works of K. p. Lundin [3], B. S. Maslov and others [4], A. S. Motorina, A.V. Bukin [5], and others.

In the process of using the soil are subjected to anthropogenic loads, resulting in changes in their agrophysical properties. On the basis of modern ideas, scientists have proposed a classification of types of anthropogenic reclamation load. The greatest destabilization of agricultural landscapes is caused by the static-destructive load [6]. In recent years, attempts have been made to generalize long-term results of observations of changes in agro-physical properties of drained hydromorphic soils in Western Siberia [5]. However, issues such as the development of these deposits, changes in their capillary and filtration properties, etc.are not covered. The data obtained by the authors on the basis of lysimetric studies, and on drained arrays subject to pyrogenic processes, require correction. In visimetrics plants are hard to model soil processes corresponding natural processes in drained areas, lysimeter, due to the high thermal regime of the soil, especially the temperature regime of the active layer of peat, the presence of other assumptions, physical and chemical processes of compaction of the organic matter occur in 1.1 to 1.3 times faster than under natural conditions, drained array. For this reason, such experimental data can practically only be used to approximate the probabilistic changes in a particular characteristic in the active layer of a peat Deposit. The calculation of the parameters of reclamation complexes should be based on soil indicators that reflect the actual natural conditions as realistically as possible.

Drainage of hydromorphic soils with subsequent plowing, processing, fertilization and crop rotation leads to a restructuring of both the water regime of the drained layer and the intensity of the soil formation process occurring in it.

When agromeliorative development of these soils activates the activity of microorganisms, which leads to increased mineralization of organic matter of peat and the accumulation of humus substances. Processes of peat formation are replaced by processes of peat decomposition and deep transformation of its organic and mineral parts. This leads to a significant change in the composition, agrophysical and chemical properties of the developed hydromorphic soils. The most significant changes in the agrophysical properties of the upper layers of the Deposit. A number of researchers divide them into basic (soil density, density of the solid phase of the soil, porosity) and functional (water and air). We follow the same gradation in our research.

Analysis of changes in agrophysical properties in the depth of peat, depending on the water supply of swamps, peat generators, etc. shows that they are closely related to the conditions of soil formation, that is, their Genesis. In addition, it is necessary to take into account the large impact of drainage reclamation. 
It should be noted that the same physical and mechanical processes occur in organogenic soils of swamps with soil - pressure nutrition as in peat soils with atmospheric type of water nutrition, but with a lower intensity. The presence of soil-pressure feed contributed to the values of ash content of peat, reaching 7.2-7.4\% for a half-meter layer of soil before laying the experiment, in the lower soil horizons of $0.6-1 \mathrm{~m}$, this indicator was $5.8-6.2 \%$. while for peat soils with an atmospheric type of water supply, the ash content did not exceed $6-6,2 \%$ and $4.7-5.2 \%$, respectively. The increased ash content of peatland in a swampy massif with a ground-pressure feed is also explained by the higher mineralization of organic matter.

Comparing the quantitative characteristics of the degree of decomposition of the organic component of peat, it can be noted that in the upper horizons of the Deposit, its value reached $35-45 \%$, within the inert layer $15-30 \%$. This is higher than the degree of decomposition of peatlands with atmospheric-slope water supply by an average of 3-5\%. During the twentyfive-year period of drainage and agricultural use of hydromorphic soils, the degree of decomposition of peat in the half-meter layer increased to $41-52 \%$, respectively, and the ash content increased by $2.1-2.6 \%$. Under the influence of peat Deposit precipitation and biochemical processes, the main soil characteristics change. The maximum increase in soil density was observed in the first years after drainage (table 1,2). Due to the fact that the swamp area of the hospital was extensively drained for 13 years by an open network of channels and the main physical processes in the peat occurred, the further dynamics of the soil density is not expressed significantly. During the research period (1978-20017), its value increased by $0.03 \mathrm{~g} / \mathrm{cm}^{3}$ and $0.02 \mathrm{~g} / \mathrm{cm}^{3}$, respectively, in the half-meter and meter layer of the active zone of the peat Deposit (table 1). Theoretical and laboratory studies have shown that there is a correlation between the density and ash content of the considered horizons of the drained peat Deposit, characterized by correlation coefficients equal to $0.68-0.76$. This relationship is approximated by a linear equation of the form:

$$
\gamma=0,03 \mathrm{~A}-0,06
$$

where: $\gamma$ - peat density, $\mathrm{g} / \mathrm{cm}^{3}, \mathrm{~A}$-ash content, $\%$

The analysis of quantitative characteristics of the density of the solid phase of the soil (table 1,2$)$ also notes a slight variation over time. If this indicator for the root zone was 1.54 $\mathrm{g} / \mathrm{cm}^{3}$ before the experiment was started, then after twenty-five years the increase was 0.03 $\mathrm{g} / \mathrm{cm}^{3}$. The density of the solid phase and the meter layer of peat increased by almost the same amount. The slight variability in time of the main physical characteristics of the peat soil on the average peat is explained by the increased moisture content of the peat Deposit in the experimental fields and its rational agricultural use (perennial grasses were grown). The presence of such conditions also contributed to the preservation of increased porosity and moisture capacity of the active layer in comparison with similar indicators of hydromorphic peat soil with an atmospheric-slope type of water supply (table 1,2). Thus, over the entire observation period, the total well capacity of the root zone of the soil decreased by 1.9 and $2.8 \%$, respectively. During the same time, the dynamics of aeration of the active horizon of the Deposit was $1.3 \%$ in the first case, and $3.3 \%$ in the second.

The obtained full-scale data show that when the depth of ground water increases from 0.5 $\mathrm{m}$ to $1.5 \mathrm{~m}$, the water - air and temperature conditions in the root zone of the hydromorphic Deposit significantly improve. As a result, peat shrinkage increases, the ratio of capillary and non-capillary porosity changes in favor of capillary porosity, and biochemical processes in the active layer of organic matter are activated. All this helps to increase the density of the soil and the density of the solid phase of the soil.

Summarizing the above, we can conclude that the drainage and agricultural use of hydromorphic massifs of the forest-steppe and subtaiga subzones of Western Siberia leads to a significant change in the agrophysical properties of the reclaimed thickness of the hydromorphic Deposit, the process of peat formation is replaced by the processes of 
decomposition of peat and deep transformation of its organic and mineral components. At the same time, these processes are much slower in swamps with ground - pressure feeding than in swamps with an atmospheric-slope type of water supply.

Table1. Dynamics of water-physical properties of drained peat soils on a marsh massif with groundpressure feed", 1978-2017.

\begin{tabular}{|c|c|c|c|c|c|c|c|c|c|c|}
\hline \multirow{3}{*}{ 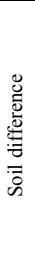 } & \multirow{3}{*}{ 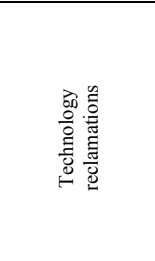 } & \multirow[b]{2}{*}{ 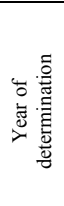 } & \multirow[b]{2}{*}{ 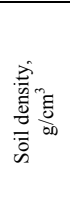 } & \multirow{2}{*}{ 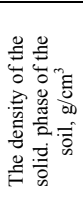 } & \multicolumn{3}{|c|}{ Duty cycle, $\%$} & \multicolumn{2}{|c|}{$\begin{array}{c}\text { Moisture } \\
\text { capacity, \% }\end{array}$} & \multirow[b]{2}{*}{ 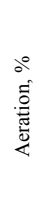 } \\
\hline & & & & & 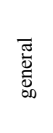 & 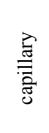 & 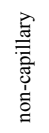 & 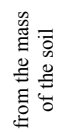 & $\begin{array}{l}\stackrel{\Xi}{\Xi} \\
\stackrel{\Xi}{0} \\
\Xi \\
0 \\
\Xi\end{array}$ & \\
\hline & & \multicolumn{9}{|c|}{ For layer $0-0.5 \mathrm{~m}$} \\
\hline \multirow{10}{*}{ 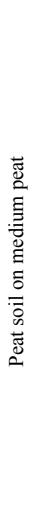 } & Of virgin swamp & 1965 & 0.124 & 1.44 & 91.9 & 60.3 & 39.7 & 630.6 & 78.2 & 13.7 \\
\hline & \multirow{9}{*}{ With draining } & 1978 & 0.162 & 1.54 & 89.5 & 86.6 & 13.4 & 406.9 & 66.4 & 24.4 \\
\hline & & 1988 & 0.187 & 1.56 & 87.6 & 93.8 & 6.2 & 344.9 & 64.5 & 23.1 \\
\hline & & 2003 & 0.197 & 1.57 & 86.4 & 96.3 & 3.7 & 323.3 & 63.7 & 22.6 \\
\hline & & 2017 & 0.202 & 1.58 & 82.6 & 97.1 & 2.9 & 312.8 & 63.2 & 22.3 \\
\hline & & \multicolumn{9}{|c|}{ For layer $0-1.0 \mathrm{~m}$} \\
\hline & & 1978 & 0.143 & 1.44 & 90.1 & 83.5 & 16.5 & 472.0 & 67.5 & 22.6 \\
\hline & & 1988 & 0.154 & 1.46 & 88.3 & 88.7 & 11.3 & 426.0 & 65.6 & 22.7 \\
\hline & & 2003 & 0.163 & 1.47 & 87.5 & 92.1 & 7.9 & 396.9 & 64.5 & 22.1 \\
\hline & & 2017 & 0.168 & 1.48 & 86.8 & 93.7 & 6.3 & 374.3 & 62.9 & 21.4 \\
\hline
\end{tabular}

Note: the total duty cycle - $\%$ of the volume; capillary and noncapillary - $\%$ of the total duty cycle.

The most significant changes in the main soil characteristics are observed in the root layer and occur in the first 2-3 years after after drying, the addition density increases by 44-48.2 $\%$. In the lower layers of the aeration zone, up to a depth of $0.8-0.9 \mathrm{~m}$, the soil density increases by $22-28 \%$. In the future, the process of changing the properties of the soil layers of the core slows down. When using drained hydromorphic peat soils for perennial grasses over a twenty-year period, the density of $0.5 \mathrm{~m}$ of the peat layer increased by 17 and $35 \%$, respectively, under the ground-pressure and atmospheric - slope type of water supply of swamps. The increase in organic mass density on small peat occurs 1.3-1.5 times faster in comparison with hydromorphic peat soils on medium peat.

No significant changes in the density of the solid phase of the soil, over time, in the upper and lower layers of medium-sized peat were found. The maximum increase in the root zone does not exceed $6.5 \%$ and reaches values of 1.58 and $1.48 \mathrm{~g} / \mathrm{cm}^{3}$, respectively, in the layer $0-0.5 \mathrm{~m}$ and $0-1.0 \mathrm{~m}$.

Under the influence of fallowing and cultivation of row crops, the quantitative values of the main physical characteristics of organic matter increase in comparison with hydromorphic soils occupied by perennial grasses.

The intensity of decomposition of organic matter increases by 4-6 times, the exchange and hydrolytic acidity increases, the degree of saturation with bases decreases, and the density of the soil in the arable layer increases 10-12 times faster. 
Table 2. Dynamics of water-physical properties of drained peat soils in a layer of 0-0.5 $\mathrm{m}$ on a swamp massif with atmospheric - slope food." 1977-2017 years.

\begin{tabular}{|c|c|c|c|c|c|c|c|c|c|c|}
\hline \multirow{2}{*}{ 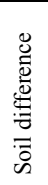 } & \multirow[b]{2}{*}{ 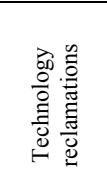 } & \multirow[b]{2}{*}{ 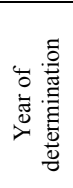 } & \multirow{2}{*}{ 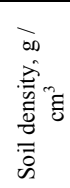 } & \multirow{2}{*}{ 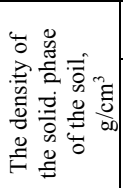 } & \multicolumn{3}{|c|}{ Duty cycle, $\%$} & \multicolumn{2}{|c|}{$\begin{array}{c}\text { Moisture capacity, } \\
\% \%\end{array}$} & \multirow[b]{2}{*}{ 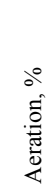 } \\
\hline & & & & & $\begin{array}{l}\overline{\widetilde{J}} \\
\overline{\vec{J}} \\
\text { 品 }\end{array}$ & $\stackrel{\vec{\Xi}}{\stackrel{\vec{Z}}{\bar{Z}}}$ & 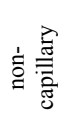 & 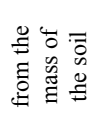 & ‡ & \\
\hline \multirow{5}{*}{ 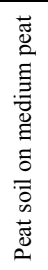 } & $\begin{array}{l}\text { Of virgin } \\
\text { swamp }\end{array}$ & 1977 & 0.119 & 1.31 & 93.4 & 53.7 & 46.3 & 662.2 & 78.8 & 14.6 \\
\hline & \multirow{4}{*}{$\begin{array}{c}\text { With } \\
\text { draining }\end{array}$} & 1980 & 0.167 & 1.57 & 89.1 & 83.3 & 16.7 & 362.9 & 60.6 & 28.5 \\
\hline & & 1990 & 0.202 & 1.61 & 86.3 & 91.2 & 8.8 & 302.5 & 61.1 & 25.2 \\
\hline & & 2000 & 0.226 & 1.63 & 84.4 & 94.8 & 5.2 & 267.3 & 60.4 & 24.0 \\
\hline & & 2017 & 0.245 & 1.65 & 82.3 & 96.2 & 3.8 & 244.8 & 59.9 & 23.4 \\
\hline \multirow{4}{*}{ 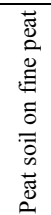 } & \multirow{4}{*}{$\begin{array}{c}\text { With } \\
\text { draining }\end{array}$} & 1980 & 0.168 & 1.59 & 89.1 & 93.7 & 6.3 & 383.9 & 64.5 & 24.8 \\
\hline & & 1990 & 0.256 & 1.79 & 83.8 & 95.0 & 5.0 & 246.1 & 63.0 & 20.2 \\
\hline & & 2000 & 0.293 & 1.88 & 80.0 & 95.8 & 4.2 & 210.9 & 61.8 & 17.4 \\
\hline & & 2017 & 0.349 & 1.93 & 78.5 & 96.2 & 3.8 & 174.8 & 61.0 & 16.4 \\
\hline
\end{tabular}

Note: the total duty cycle - \% of the volume; capillary and noncapillary - \% of the total duty cycle.

The maximum reduction in soil borehole is observed at the initial stages of development of marsh complexes and reaches $4.3 \%$. In the future, this process slows down and over the past seventeen years in a layer of $0-0.5 \mathrm{~m}$, was 2.1 and $3.8 \%$, respectively, for hydromorphic with atmospheric slope and soil-pressure type of nutrition. The total borehole in the upper soil horizons is gradually approaching the borehole of the lower layers of the Deposit. This increases the number of capillary pores and reduces the proportion of aeration pores. Changing the ratio of capillary and non-capillary wells leads to a decrease in soil moisture capacity. The intensity of drainage and precipitation of the peat Deposit has a significant impact on the reduction of moisture capacity. The dynamics of moisture content of peat in agricultural use and mineralization of organic matter is insignificant. Over the twenty-year period, its value decreased by $2.7-3 \%$.

Reclamation of soils in swamp geosystems leads to significant changes in the degree of decomposition of organic matter and its ash content. During the study period, the degree of decomposition of organogenic soils in the half-meter layer increased by $4-6 \%$, reaching 41 $-52 \%$, and the ash content increased by $2.1-2.6 \%$, respectively. The values of the considered indicators are $3-5 \%$ lower on wetlands with atmospheric - slope type of water supply.

In connection with the above, the rationale for the rational use and protection of hydromorphic marsh landscapes, the design of reclamation measures on them, must be carried out taking into account the features and specifics of natural conditions and using the values of agrophysical characteristics of peat deposits, which actually reflect their state in the future, otherwise it is possible either under-draining or over-draining of soils.

\section{Conclusions}

It has been determined that:

In the process of evolution, as well as under conditions of anthropogenic impact, hydromorphic soils of natural-territorial complexes, their properties undergo significant changes. The process of peat formation is replaced by the processes of decomposition of peat and the deep transformation of its organic and mineral components. New hydromorphic geosystems are being formed. 
The most significant changes in the main soil characteristics are noted in the rootinhabited layer and occur in the first 2-3 years after drainage, the density of addition increases by $44-48.2 \%$. In the lower layers of the aeration zone, to a depth of $0.8-0.9 \mathrm{~m}$, the soil density increases by $22-28 \%$. Subsequently, the process of changing the properties of soil layers in the core slows down. At the same time, these processes are much slower in swamps with soil - pressure feeding than in swamps with atmospheric - slope type of water supply.

An increase in the density of organic matter on shallow peat occurs 1.3-1.5 times faster in comparison with hydromorphic peat soils on medium peat. Significant changes in the density of the solid phase of the soil, over time (over 38 years), in the upper and lower layers of medium-thick peat have not been established. The maximum increase in this indicator in the root zone does not exceed $6.5 \%$ and reaches a value of 1.58 and $1.48 \mathrm{~g} / \mathrm{cm}^{3}$, respectively, in a layer of $0-0.5 \mathrm{~m}$ and $0-1.0 \mathrm{~m}$.

Under the influence of steaming and cultivation of row crops, the quantitative values of the basic physical characteristics of organic matter increase in comparison with hydromorphic soils occupied by perennial grasses. The decomposition rate of organic matter increases by $4-6$ times, the exchange and hydrolytic acidity increase, the degree of saturation with bases decreases, the soil density in the arable layer increases 10-12 times faster.

In lysimetric installations, the physical and chemical processes of compaction of organic matter occur 1.1-1.3 times faster than under natural conditions of the drained massif; therefore, it is not correct to use such results when developing land reclamation projects.

\section{References}

1. M.I. Neustadt, Scientific prerequisites for the development of swamps in Western Siberia (Nauka Publishing house, Moscow, 1977)

2. V. N. Efimov, Peat soils and their fertility (Agropromizdat, Leningrad, 1986)

3. K. P. Lundin, Water properties of a peat Deposit (Vintage, Minsk, 1964)

4. B. S. Maslov, I. V. Minaev, Drainage systems of the XXI century (Russian Agricultural Academy, Moscow, 1999)

5. A. S. Motorin, A.V. Bukin, Agriculture and chemization 47(5) (2017)

6. P. I. Pylenok, I. V. Sidorov, Environmental reclamation regimes and technologies (Russian Agricultural Academy, Moscow, 2004) 